\title{
Synthesis and Electrochemical Characterization of Polypyrrole/Multi-walled Carbon Nanotube Composite Electrodes for Supercapacitor Applications
}

\author{
Santhosh Paul, Yoon-Sung Lee, Ji-Ae Choi, Yun Chan Kang, ${ }^{\dagger}$ and Dong-Won Kim* \\ Department of Chemical Engineering, Hanyang University, Seoul 133-791, Korea. *E-mail: dongwonkim@hanyang.ac.kr \\ ${ }^{\dagger}$ Department of Chemical Engineering, Konkuk University, Seoul 143-701, Korea \\ Received January 29, 2010, Accepted March 4, 2010
}

\begin{abstract}
The nanocomposites of polypyrrole (PPy) and multi-walled carbon nanotube (MWCNT) with different composition are synthesized by the chemical oxidative polymerization method. In these composites, the MWCNTs are uniformly coated by PPy with different thickness. The electrochemical properties of the composite electrodes are investigated by cyclic voltammetry, galvanostatic charge-discharge cycling and electrochemical impedance spectroscopy. The full cells assembled with the PPy/MWCNT composite electrodes deliver initial specific capacitances ranging from 146.3 to $167.2 \mathrm{~F} / \mathrm{g}$ at $0.5 \mathrm{~mA} / \mathrm{cm}^{2}$ and exhibit stable cycling characteristics. The effect of content of MWCNT in the composite on cycling performance of the cells is also investigated.
\end{abstract}

Key Words: Cycling performance, Multi-walled carbon nanotubes, Nanocomposite, Polypyrrole, Supercapacitor

\section{Introduction}

The growing demand for portable electronic systems, digital communication devices and electric vehicles has prompted considerable interest in supercapacitors with high power density and long cycle life. ${ }^{1-3}$ The major types of materials used in supercapacitors are: (i) high surface area activated carbons, (ii) redox metal oxides and (iii) conducting polymers. ${ }^{46}$ Among them, conducting polymers attracted great deal of interest due to their high specific capacitance, low material cost, easy synthesis and many other inherent properties. ${ }^{7-14}$ However, the main drawback of conducting polymer-based supercapacitors is poor cycle life due to the volume change during doping and dedoping process. Hence it is necessary to strengthen adequately the electrochemically active sites of conducting polymers by the addition of large surface area carbon materials or carbon nanotubes (CNTs). CNTs have been attractive materials for electrodes of supercapacitors due to their high accessible surface area, high electronic conductivity, chemical and mechanical stability. In order to improve the mechanical and electrochemical properties of electrodes based on conducting polymers, the composites of conducting polymers and CNTs have been synthesized for supercapacitor applications. ${ }^{15-22}$ It has been shown that CNTs play a role of a perfect backbone for a homogeneous distribution of conducting polymer in the composites. Recently, Kim et al. reported that high-specific capacitance and good high-rate capability of polypyrrole(PPy)/CNT composite electrodes could be achieved by controlling pore size in a three-dimensional entangled structure of a CNT film. ${ }^{22}$

In this work, we tried to improve the cycling stability of the cell without significant drop in specific capacitance. With the aim of improving the cycleability of the PPy-based electrode, we synthesized PPy/multi-walled carbon nanotube (MWCNT) composites with different composition by the chemical oxidative polymerization method. In this study, the electrochemical properties of the PPy/MWCNT nanocomposite electrodes were investigated by cyclic voltammetry, galvanostatic charge-discharge cycling and electrochemical impedance spectroscopy. The effect of content of MWCNT in the composite on electrochemical performance of the cells was also investigated.

\section{Experimental}

Synthesis of PPy/MWCNT composites. Pyrrole, anhydrous ferric chloride $\left(\mathrm{FeCl}_{3}\right)$, poly (vinyl pyrrolidone) (PVP), polytetrafluoroethylene (PTFE), isopropanol (IPA), N-methylpyrrolidone (NMP) and potassium chloride $(\mathrm{KCl})$ were purchased from Aldrich chemicals. Pyrrole was purified before reaction and rest of the chemicals were used as received. MWCNTs (Hanwha Nanotech) prepared by chemical vapor deposition were used without further purification. The PPy/MWCNT composites were synthesized by the conventional oxidative chemical polymerization method using anhydrous $\mathrm{FeCl}_{3}$. The content of reactants for synthesizing PPy/MWCNT composites with different composition is given in Table 1. Different quantity of MWCNTs $(0.100,0.210,0.325 \mathrm{~g})$ was stirred vigorously in $100 \mathrm{~mL}$ aqueous $\mathrm{FeCl}_{3}$ solution. This suspension was sonicated for $3 \mathrm{~h}$, to facilitate the good dispersion of MWCNTs. Pyrrole $(0.967 \mathrm{~g})$ was added very slowly and drop wise into the stirring solution. The polymerization was allowed to continue for another $4 \mathrm{~h}$. The precipitated composite material was then filtered, washed with water and methanol. It was vacuum dried at $70{ }^{\circ} \mathrm{C}$ overnight. From the measurements of weight of PPy/MWCNT

Table 1. Summary of reactant composition for synthesizing PPy/ MWCNT composites.

\begin{tabular}{cccc}
\hline & PCNT-10 & PCNT-20 & PCNT-30 \\
\hline MWCNT $(\mathrm{g})$ & 0.100 & 0.210 & 0.325 \\
FeCl $_{3}(\mathrm{~g})$ & 3.880 & 3.880 & 3.880 \\
Pyrrole $(\mathrm{g})$ & 0.967 & 0.967 & 0.967 \\
Water $(\mathrm{mL})$ & 100 & 100 & 100 \\
\hline
\end{tabular}


composites, the content of MWCNT was calculated to be 9.6, $20.1,30.5$ by weight for three composite materials, respectively. The PPy/MWCNT composites synthesized are designated PCNT-10, PCNT-20 and PCNT-30 according to the content of MWCNT in the composites.

Electrode preparation and cell assembly. Electrode was prepared by coating a IPA/NMP-based slurry of composite material, super-P, PTFE and PVP (75:15:7:3 by weight) on a Ti foil. The excellent water wetting and adhesive properties of PVP have been well known and thus a proper amount of PVP was added along with PTFE as a binder. The binder materials (PTFE/PVP) were first dissolved in IPA/NMP mixed solvent and then PPy/MWCNT composite and super-P powder were added into the polymer binder solution. The solution was sonicated for $1 \mathrm{~h}$ and ball-milled for another $15 \mathrm{~h}$ in order to make a homogenous slurry. The resultant slurry was cast on to Ti foil by using a doctor blade. The electrodes were dried in a vacuum oven at $80{ }^{\circ} \mathrm{C}$ for $24 \mathrm{~h}$, and roll-pressed to enhance particulate contact and adhesion to the foil. Sandwich-type cells were assembled with two symmetric PPy/MWCNT electrodes (area: $1.0 \mathrm{~cm}^{2}$ ). The electrolyte used in assembling the cell was $1.0 \mathrm{M}$ $\mathrm{KCl}$ aqueous electrolyte solution with paper separator (thickness: $40 \mu \mathrm{m}$, Nippon Kodoshi Co.). Both electrodes and separator were soaked in the electrolyte before the cell assembly. The symmetrical cell was enclosed in a metallized plastic bag and vacuum-sealed.

Characterization and electrochemical analysis. Morphologies of pristine MWCNTs and PPy/MWCNT composites were examined using a field emission scanning electron microscopy (FESEM, JEOL JSM-6701). Cyclic voltammograms (CVs) were recorded from 0 to $0.6 \mathrm{~V}$ at different scan rates. AC impedance measurements were performed on $\mathrm{CH}$ Instrument in the frequency range of $0.1 \mathrm{~Hz}-100 \mathrm{kHz}$ with an amplitude of $10 \mathrm{mV}$. Galvanostatic charge-discharge cycling of the cell was conducted over voltage ranges of $0-0.6 \mathrm{~V}$ with battery-test equipment at constant current densities from 0.5 to $10 \mathrm{~mA} / \mathrm{cm}^{2}$ at room temperature.

\section{Results and Discussion}

The FESEM images of the pristine MWCNTs and various PPy/MWCNT composites are shown in Figure 1. The average diameter of the pristine nanotubes (Figure 1 (a)) was measured to be about $40 \mathrm{~nm}$. From the images of PPy/MWCNT composites in Figure 1 (b)-(d), it can be seen that all the composites exhibit well dispersed carbon nanotubes enwrapped uniformly with PPy. This suggests that the interaction between polymer molecules and MWCNTs overcomes the Van der Waals interaction between MWCNTs, which generally otherwise would result in separate growth or aggregates of PPy. As the content of MWCNT increases in the composite, a gradual decrease of thickness of the PPy layer over nanotubes is clearly observed. As shown in Figure (b)-(d), the average diameter of MWCNT in the composites increases to about 77, 58, $51 \mathrm{~nm}$ and thus the thickness of the PPy coating is estimated to be 18.5, 9.0, $5.5 \mathrm{~nm}$ for PCNT-10, PCNT-20, PCNT-30, respectively. In these composites, MWCNTs can offer good mechanical support to PPy and also ensure good electronic conduction in the electrode. The electrode film cast on Ti foil exhibits highly interconnected and porous morphology, as shown in Figure 1 (e), which is highly desirable for the fast ion diffusion and migration in the electrodes. The co-addition of PVP as a binder material could also help in opening many channels for the aqueous electrolyte to pass through and it gave an improved wetting of the electrode material with aqueous electrolyte.

The electrochemical performance of the PPy/MWCNT composite electrode was evaluated by the $\mathrm{CVs}$ of the two-electrode cells assembled with the same electrode. The voltammograms obtained for the PCNT-20 composite electrode at different scan rates are presented in Figure 2. The CVs obtained for the other two composite electrodes also followed the same behavior as shown in Figure 2. The CVs of the composite electrode look almost like rectangular shape with good symmetry at all scan rates, showing highly efficient capacitive behavior with good charge propagation. CVs shown in Figure 2 are very similar to
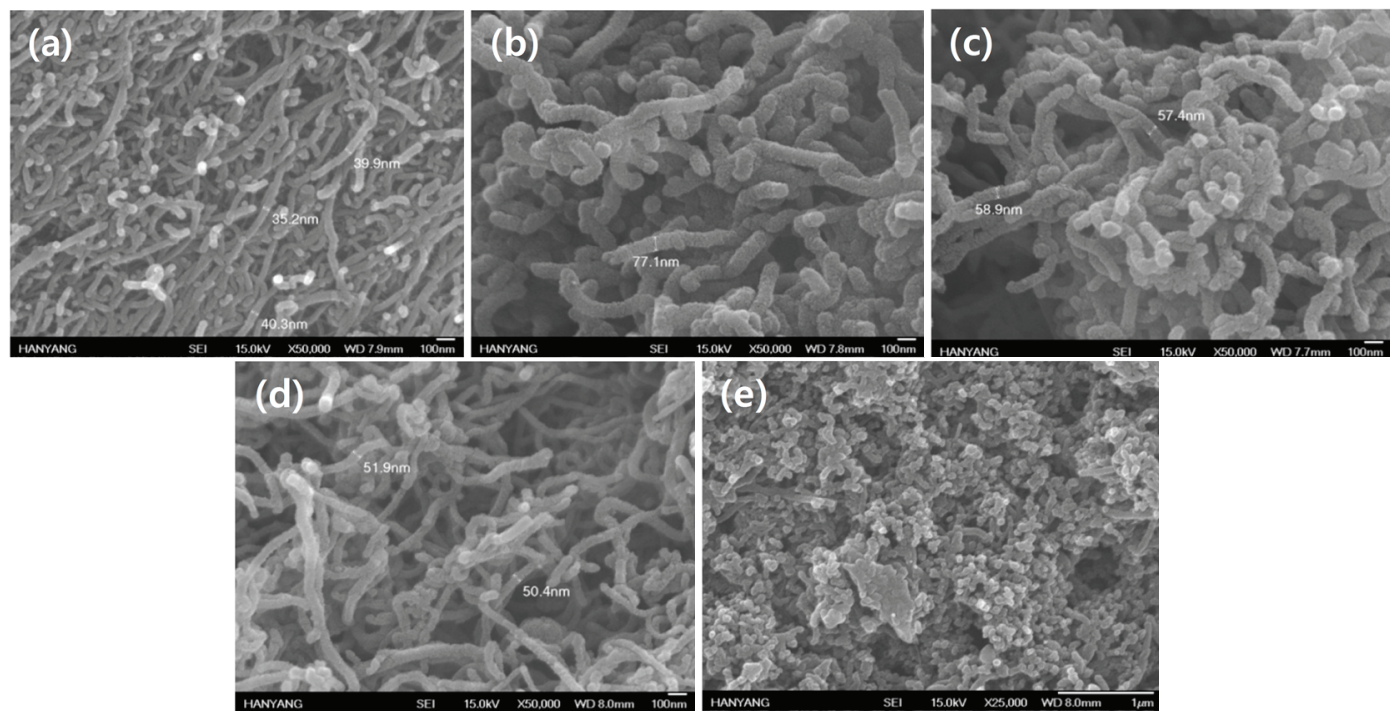

Figure 1. FESEM images of (a) pristine MWCNTs, (b) PCNT-10, (c) PCNT-20, (d) PCNT-30 and (e) composite electrode film cast on Ti foil. 


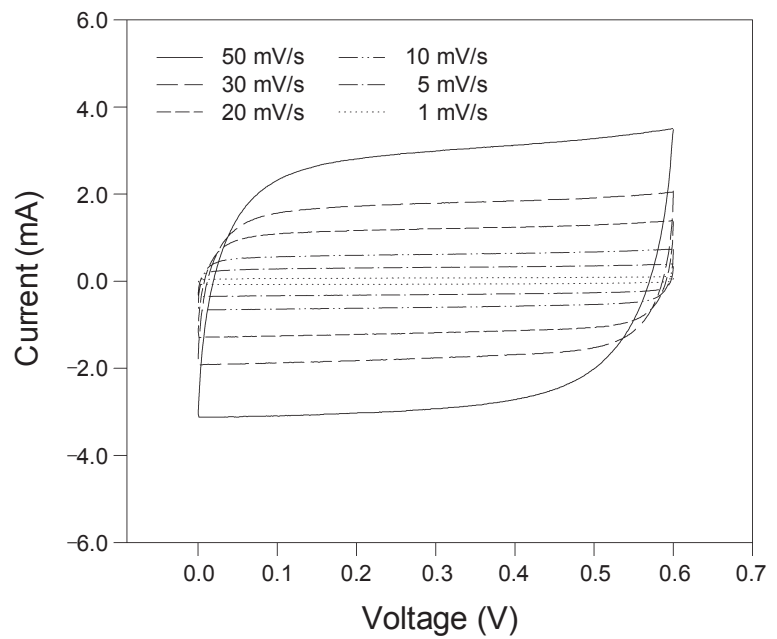

Figure 2. Cyclic voltammograms obtained for the symmetric twoelectrode cell assembled with PCNT-20 composite. (electrolyte: $1.0 \mathrm{M}$ $\mathrm{KCl})$.

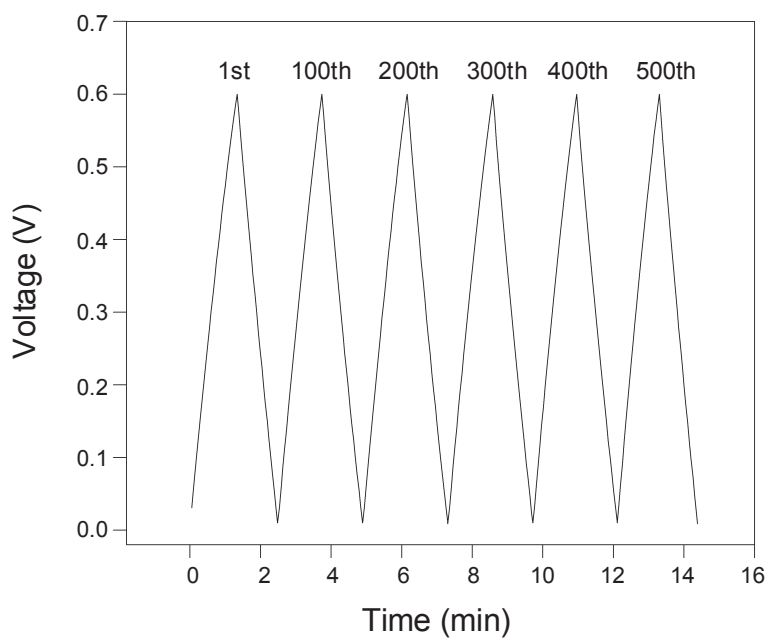

Figure 3. Charge and discharge curves of the symmetric cell assembled with PCNT-20 composite electrode.

those observed in the other PPy/CNT composite electrodes. ${ }^{17,20-23}$ These composite electrodes exhibited no significant change in CV shape even after 500 cycles, which indicates good cycleability of the PPy/CNT electrodes.

The symmetric cell assembled with PCNT-20 composite electrode was subjected to charge-discharge cycling in the voltage range of $0-0.6 \mathrm{~V}$ at a constant current of $0.5 \mathrm{~mA} / \mathrm{cm}^{2}$, and its charge-discharge curves are shown in Figure 3. These curves are observed to be perfectly linear and there is no significant IR drop, which indicates prominent capacitive behavior of the cell. The charging and discharging time for the cell is maintained to be constant throughout the cycling, which demonstrates that the PPy/MWCNT composite electrode has high stability. The supercapacitors based on PPy composites experienced either very fast or gradual decrease in specific capacitance during repeated cycling, as previously reported. ${ }^{15,17}$ The specific capacitance of the electrode was calculated using the formula of $C(\mathrm{~F} / \mathrm{g})=2 I t /(m \Delta E)$ where, $I$ is the current applied for the charging and discharging, $t$ is the time of discharge, $\Delta E$ is the

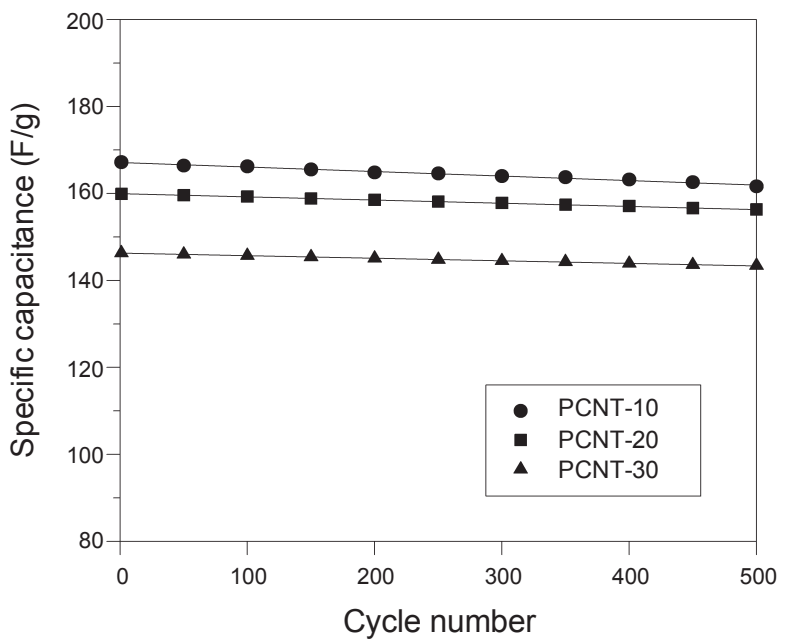

Figure 4. Specific discharge capacitance as a function of cycle number for the cells assembled with three different composite electrodes, which are obtained at a constant current density of $0.5 \mathrm{~mA} / \mathrm{cm}^{2}$.

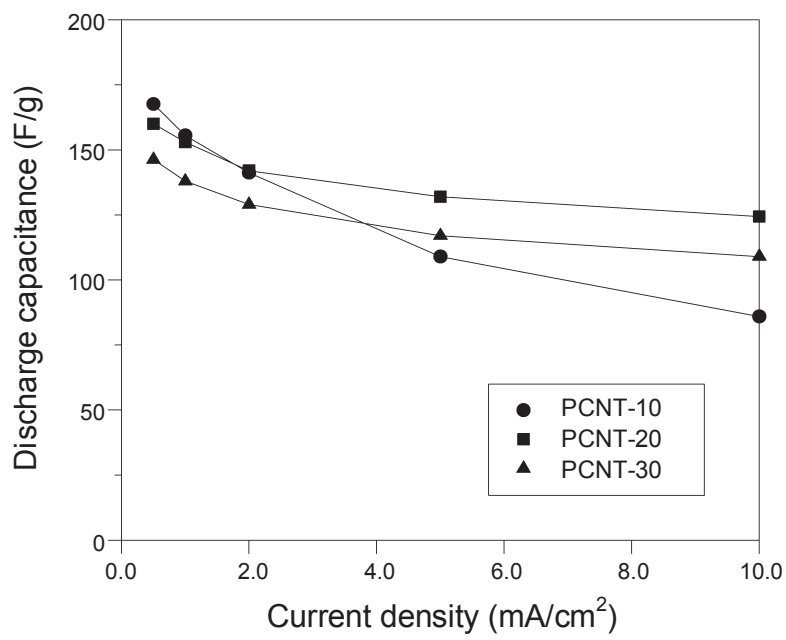

Figure 5. Specific capacitance as a function of current density for the cells assembled with different PPy/MWCNT composite electrodes.

voltage difference between the upper and lower potential limit and $m$ is the mass of active materials (PPy and MWCNT) in one of the electrodes. The factor of " 2 " arises from the fact that the total capacitance measured from the cell is the addition of two equivalent single electrode capacitors in series. ${ }^{17}$ The discharge capacitance obtained for all the cells assembled with different composite electrodes are shown in Figure 4. The initial specific capacitances obtained from the galvanostatic chargedischarge measurements ranges from 146.3 to $167.2 \mathrm{~F} / \mathrm{g}$, and are found to be increased with increasing PPy content. It should be noted that pure MWCNT has very low specific capacitance not exceeding $40 \mathrm{~F} / \mathrm{g}$. An increase in specific capacitance of PPy/MWCNT composite with increasing PPy content can be thus associated with pseudo capacitance contributing from PPy. It is also found that the loss of capacitance is little during the charge and discharge cycles. This result suggests that the PPy/ MWCNT composite electrodes are very stable under charge and discharge cycles and the active electrode materials are keeping good interfacial contacts in the electrode. The data given 


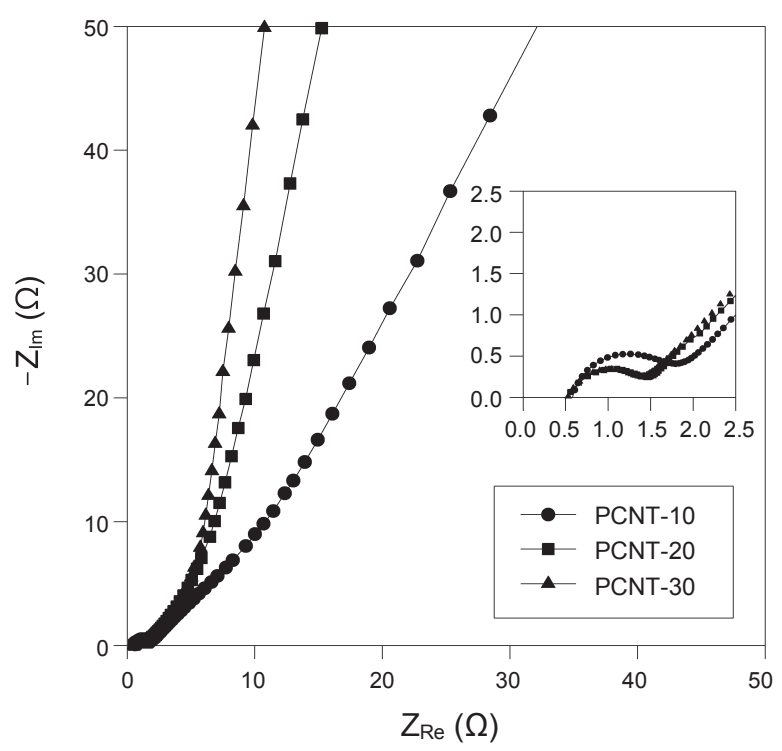

Figure 6. AC impedance spectra of the cells assembled with different $\mathrm{PPy} / \mathrm{MWCNT}$ composite electrode, which are obtained after 500 cycles in the voltage range of $0-0.6 \mathrm{~V}$.

in Figure 4 show that the cell assembled with PCNT-30 has the best cycleability among the cells considered in this work.

Rate capabilities of cells assembled with PPy/MWCNT electrodes were evaluated with varying current densities from 0.5 to $10 \mathrm{~mA} / \mathrm{cm}^{2}$, which are shown in Figure 5. As expected, a decrease in capacitance is observed with increasing the current densities. The cell assembled with PCNT-20 composite has the highest capacitance at high current rates. As compared, the capacitance drop is the most prominent in the cell of PCNT-10 composite electrode. The reduction of capacitance in the cell of PCNT-10 at high current rate may be associated with PPy layer with high thickness, which gives high internal resistance of the cell, as explained later. In case of PCNT-30 electrode, the amount of PPy is not enough for giving high capacitance as compared that of the cell assembled with PCNT-20. These results suggest that there should be an optimum content of MWCNT in the composite in order to provide both high specific capacitance and good rate capability of the cell.

In order to investigate the effect of content of MWCNT on the impedance behavior of the cells, the ac impedance of the cells was measured. Figure 6 presents ac impedance spectra of the cells, which are obtained at discharged state after 500 cycles in the voltage range of $0-0.6 \mathrm{~V}$. In all the spectra, the cells displayed a semicircle followed by a capacitive spike. The intercepts of the real axis at high frequency are $0.58,0.54,0.53 \Omega$ for the cells assembled with PCNT-10, PCNT-20 and PCNT-30, respectively. This intercept is known to be related to the intrinsic resistance of the active materials, the electrolyte resistance, the electrical leads, and the contact resistance at the interface of active materials/current collector. ${ }^{24}$ A slight decrease in high frequency-resistance with increasing content of MWCNT may be due to the decrease in resistance of the active materials. On the other hand, the semicircle appeared at middle-to-low frequency region is related to the charge transfer reaction occurring in the cells. Charge transfer resistances calculated from these spectra are $1.21,0.90$ and $0.85 \Omega$ for the cells assembled with PCNT-10, PCNT-20 and PCNT-30, respectively. A thin coating of PPy on MWCNT can facilitate an effective penetration of electrolyte into PPy, leading to enhanced participation of active materials in the charge transfer reaction, which results in decrease of charge transfer resistance with increasing MWCNT content in the PPy/MWCNT electrode. It suggests that the introduction of proper content of MWCNTs into the composite may facilitate the charge transfer and reduce the internal resistance of the electrode.

\section{Conclusions}

The homogeneous coating of PPy over MWCNTs could be achieved by the simple chemical oxidative polymerization method. Stable cycling could be achieved for the cells fabricated with these composites during the galvanostatic charge-discharge tests. The performance of the cells depended mainly on the content of MWCNT in the composite electrode. The cell assembled with PCNT-10 showed the highest specific capacitance due to the higher content of PPy. In consideration of cycling stability and rate capability, the cells based on PCNT-20 and PCNT-30 exhibited better performance. From these results, the PPy/MW CNT composites with optimum composition could be considered as promising electrode materials in the application of supercapacitors with high capacitance and long cycle life.

Acknowledgments. This work was supported by the National Research Foundation of Korea (NRF) grant funded by the Korea government (MEST)(No. 2009-0071851). This work is also the outcome of a Manpower Development Program for Energy \& Resources supported by the Ministry of Knowledge and Economy (MKE).

\section{References}

1. Conway, B. E. J. Electrochem. Soc. 1991, 138, 1539.

2. Frackowiak, E.; Beguin, F. Carbon 2002, 40, 1775.

3. Arico, A. S.; Bruce, P.; Scrosati, B.; Tarascon, J. M.; Schalkwijk, W. V. Nat. Mater. 2005, 4, 366.

4. Kim, T.; Ham, C.; Rhee, C. K.; Yoon, S. H.; Tsuji, M.; Mochida, I. Carbon 2009, 47, 226.

5. Sarangapani, S.; Tilak, B. V.; Chen, C. P. J. Electrochem. Soc. 1996, 143,3791 .

6. Park, G. J.; Kalpana, D.; Kumar, A.; Nakamura, H.; Lee, Y. S.; Yoshio, M. Bull. Korean Chem. Soc. 2009, 30, 817.

7. Jurewicz, K.; Delpeux, S.; Bertagna, V.; Beguin, F.; Frackowiak, E. Chem. Phys. Lett. 2001, 347, 36.

8. Park, J. H.; Ko, J. M.; Park, O. O.; Kim, D. W. J. Power Sources 2002, 103, 20.

9. Ryu, K. S.; Kim, K. M.; Park, N. G.; Park, Y. J.; Chang, S. H. J. Power Sources 2002, 103, 305.

10. Ryu, K. S.; Lee, Y. G.; Hong, Y. S.; Park, Y. J.; Wu, X.; Kim, K. M.; Kang, M. G.; Park, N. G.; Chang, S. H. Electrochim. Acta 2004, 50,843 .

11. Kim, J. H.; Lee, Y. S.; Sharma, A. K.; Liu, C. G. Electrochim. Acta 2006, 52, 1727.

12. Fan, L. Z.; Maier, J. Electrochem. Commun. 2006, 8, 937.

13. Kim, B. C.; Ko, J. M.; Wallace, G. G. J. Power Sources 2008, 177, 665.

14. Kim, B. C.; Kwon, J. S.; Ko, J. M.; Park, J. H.; Too, C. O.; Wallace, 
G. G. Synth. Met. 2010, 160, 94.

15. An, K. H.; Jeon, K. K.; Heo, J. K.; Lim, S. C.; Bae, D. J.; Lee, Y. H. J. Electrochem. Soc. 2002, 149, A1058.

16. Xiao, Q.; Zhou, X. Electrochim. Acta 2003, 48, 575.

17. Khomenko, V.; Frackowiak, E.; Beguin, F. Electrochim. Acta 2005, 50, 2499.

18. Sivakkumar, S. R.; Kim, W. J.; Choi, J. A.; MacFarlane, D. R.; Forsyth, M.; Kim, D. W. J. Power Sources 2007, 171, 1062.

19. Sivakkumar, S. R.; Ko, J. M.; Kim, D. Y.; Kim, B. C.; Wallace, G.
G. Electrochim. Acta 2007, 52, 7377

20. Oh, J.; Kozlov, M. E.; Kim, B. G.; Kim, H. K.; Baughman, R. H.; Hwang, Y. H. Synth. Met. 2008, 158, 638.

21. Lin, X.; Xu, Y. Electrochim. Acta 2008, 53, 4990.

22. Kim, J. Y.; Kim, K. H.; Kim, K. B. J. Power Sources 2008, 176, 396.

23. Xiao, Q.; Zhou, X. Electrochim. Acta 2003, 48, 575.

24. Mi, H.; Zhang, X.; Ye, X.; Yang, S. J. Power Sources 2008, 176, 403. 